\title{
Suportando a Modelagem de Processo de Monitoração e Controle em Micro e Pequenas Empresas, alinhado ao CMMI, MPS.BR e ISO/IEC15504
}

\author{
Jean Carlo Rossa Hauck ${ }^{1}$, Christiane Gresse von Wangenheim ${ }^{12}$, Marcello Thiry ${ }^{2}$ \\ ${ }^{1}$ Universidade Federal de Santa Catarina - Florianópolis - SC - Brasil. \\ ${ }^{2}$ Universidade do Vale do Itajaí - UNIVALI - LQPS - Laboratório de Qualidade e \\ Produtividade de Software da Universidade do Vale do Itajaí, Campus VII - São José - \\ $\mathrm{SC}-$ Brasil \\ jeanhauck@inf.ufsc.br, \{gresse, thiry\}@univali.br
}

\begin{abstract}
This paper presents an extension of a software process modeling approach, including a process guide, to support the software project monitoring and control process modeling and alignment to the most important reference models, adapting the modeled process to the typical characteristics and limitations of small and very small software companies. The approach, complemented by the guide, is applied in a case study and the results are also presented.
\end{abstract}

Resumo. Este artigo apresenta a extensão de uma abordagem de modelagem de processo, por meio da inclusão de um guia, para suportar a modelagem do processo de monitoração e controle de projetos de software e o seu alinhamento aos principais modelos de referência, adaptando-o às características e limitações típicas de micro e pequenas empresas de software. A abordagem resultante, complementada pelo guia, é aplicada em um estudo de caso e os resultados da sua aplicação são também apresentados.

\section{Introdução}

Existem diversos modelos de referência para a melhoria de processos de software disponíveis atualmente [SEI 06] [ISO/IEC 05] [SOFTEX 06], mas, em geral, as Micro e Pequenas Empresas ${ }^{1}$ de software (MPEs) tendem a enfrentar muitas dificuldades para produzir software com qualidade e produtividade [SEBRAE-SP 00] [Wangenheim 07] [MCT 05].

Uma das razões para este fato é que muitas MPEs simplesmente não conhecem estes modelos de referência [Storey 82] [Daft 92] [Johnson 99]. Observa-se ainda que as avaliações de conformidade a estes modelos e padrões normalmente são caras, consomem muito tempo, e, conseqüentemente, são difíceis de executar em MPEs [Paulk 98] [Rout 00]. Percebe-se também que os modelos e padrões são mais direcionados para

\footnotetext{
${ }^{1}$ Apesar de não existir uma unanimidade quanto à classificação de uma empresa como MPE, a mais comumente aceita é a classificação do SEBRAE [SEBRAE 07], que classifica como MPE empresas prestadoras de serviços com 1 a 9 funcionários [SEBRAE-SP 00].
} 
grandes organizações [MCT 05] [Daft 92] [Johnson 99] [Paulk 98], incluindo, por exemplo, práticas para a documentação e formalização do processo [SIS 06]. Estes procedimentos têm sido criticados por não serem apropriados para o contexto de MPEs [MCT 05], em sua maioria caracterizadas por processos mais informais e estruturas organizacionais [Johnson 99] focadas, primeiramente, em garantir a entrega do produto visando a sobrevivência da empresa.

Abordagens ágeis de processo de software, como [Beck 99] [Schwaber 95], que propõem processos mais dinâmicos e flexíveis para o desenvolvimento de software, tendem a serem mais voltadas à realidade das MPEs. Entretanto, estas abordagens também não trazem descrições detalhadas de suas práticas de modo que possam ser diretamente aplicadas e evidenciadas [Alegria 06] [Pikkarainen 06].

Em geral, observa-se nas propostas atuais a ausência de um auxílio mais concreto à implantação de melhoria de processos, indicando alternativas de como as práticas sugeridas pelos modelos de referência podem ser aplicadas à realidade das MPEs. Neste sentido, o LQPS - Laboratório de Qualidade e Produtividade de Software, da UNIVALI, vem trabalhando na elaboração de guias para a aplicação de melhores práticas especificamente no contexto de MPEs [Wangenheim 07] [Wangenheim 06] [Wangenheim 06b].

Além de atender às características das MPEs, para que possam ser implantadas em uma determinada organização, as práticas descritas em um guia também precisam ser adaptadas às características específicas daquela organização e alinhadas aos seus objetivos de negócio [Wangenheim 06] [Wangenheim 06b] [Hauck 04] [Weber 05] por meio da modelagem do seu processo.

Desta forma, o presente trabalho propõe uma abordagem de modelagem de processo de software, suportada por um guia alinhado aos modelos de referência CMMI-DEV, ISO/IEC 15504 e MPS.BR, que apresenta alternativas de implantação das práticas adaptadas ao contexto de MPEs. A abordagem proposta evolui a abordagem de modelagem de processo ASPE/MSC ${ }^{2}$ [Weber 05], provendo suporte concreto para a modelagem prescritiva a partir da introdução do guia. A extensão de uma abordagem de modelagem de processo como ASPE/MSC se faz necessária, pois, as abordagens de modelagem de processo atuais [Machado 00] [Scott 00] [OMG 07] [Weber 05] [Thiry 06] [Becker 01] (mesmo as que objetivam a modelagem de processos em MPEs), em geral, não fornecem informações num nível de detalhe suficiente para a modelagem de processos específicos.

Neste sentido, o presente trabalho abrange a elaboração de um guia detalhado de monitoração e controle de projetos que dá suporte à modelagem deste processo específico. O processo de monitoração e controle de projetos tem o objetivo de oferecer um entendimento do progresso do projeto, para que ações corretivas apropriadas possam ser tomadas sempre que o desempenho do projeto se desviar significativamente do seu plano [SEI 06]. O enfoque em monitoração e controle de projetos se justifica pelo fato de que as MPEs tipicamente apresentam processos bastante informais e imaturos [Wangenheim 07], onde as iniciativas de melhoria de processos têm enfocado os níveis

\footnotetext{
${ }^{2}$ Approach for Software Process Establishment in Micro and Small Companies
} 
de maturidade iniciais, como o nível 2 do CMMI-DEV V1.2 ou nível G do MPS-BR, nos quais um dos principais processos é a gerência de projetos.

Completando os modelos de referência de melhoria de processo, existem modelos, normas e guias específicos para gerência de projetos, como por exemplo [PMI 04] [ISO/IEC 97] [ANSI/EIA 98]. Ainda mais especificamente voltadas para a gerência de projetos de software, considerando as características e dificuldades específicas, como a invisibilidade e intangibilidade inerentes ao software [Acuña 00], existem abordagens de gerência que procuram estabelecer práticas para gerenciar projetos de software [Hughes 02] [Martins 06] [Galorath 05]. Entretanto, estas abordagens possuem, em certo grau, as mesmas limitações dos modelos e normas de referência por serem genéricas e não apresentarem um auxílio mais concreto de como implementar as práticas sugeridas.

Outros guias ainda fornecem diretrizes de como implementar as práticas requeridas pelos modelos de referência [Kulpa 03] [Jalote 00], incluindo a monitoração e controle de projetos. Porém, a maioria destas abordagens ou guias não é voltada a MPEs e a sua complexidade, em geral, dificulta a sua adaptação para este tipo de organização [SEBRAE-SP 00].

Desta forma, a existência de uma abordagem para modelagem do processo de monitoração e controle de projetos de software, completada por um guia que indica ferramentas e práticas especificamente adaptadas ao contexto de MPEs brasileiras, alinhado aos modelos CMMI-DEV, MPS-BR e ISO/IEC 15504, tende a aumentar a possibilidade de uma MPE implantar técnicas sistemáticas de gerência de projetos de software.

O presente artigo apresenta esta abordagem, inicialmente propondo os requisitos para um guia de projeto, em seguida detalhando a abordagem proposta e estabelecendo um guia para ser utilizado na abordagem, sendo por último apresentado um estudo de caso.

\section{Requisitos de um Guia de Processo de Monitoração e Controle para MPEs}

Um Guia de Processo de Monitoração e Controle de Projetos deve ser compatível com os principais modelos de melhoria utilizados (CMMI-DEV V1.2, MPS-BR V1.1 e ISO/IEC 15504), considerando especificamente os processos:

- Área de Processo de Monitoração e Controle do CMMI-DEV V1.2,

- Processo MAN.3 Gerência de Projetos da ISO/IEC 15504 e

- Gerência de Projetos do MPS.BR V1.1,

fornecendo um suporte eficiente e eficaz para a aplicação das práticas definidas nestes modelos. Para ser adequado à aplicação em MPEs, o guia deve também atender os seguintes requisitos [SEBRAE-SP 00] [Wangenheim 07] [MCT 05]:

- Custo: o guia deve ser livre para utilização e adaptação sem custos. Ele deve, preferencialmente, indicar somente ferramentas e técnicas livres;

- Simplicidade: as indicações descritas no guia (técnicas, processos etc.) devem ser o mais simples possível, de forma a reduzir a complexidade da implantação; 
- Facilidade de compreensão: o guia deve ser fácil de entender, não exigindo conhecimento profundo na área de gerência de projetos. Neste sentido, deve ser escrito em português e utilizar linguagem coloquial;

- Facilidade de implantação: o guia deve indicar oportunidades de automatizar o processo de monitoração e controle para reduzir o esforço necessário à implantação;

- Detalhamento: o guia deve fornecer descrições em um nível de detalhe suficiente, que torne possível a execução das atividades;

- Escopo: o guia deve fornecer suporte para todo o processo de monitoração e controle, incluindo vários artefatos que auxiliem a execução do processo, como por exemplo, descrições de processos, templates, ferramentas e cenários;

- Adaptabilidade: o guia deve facilitar sua adaptação aos diversos tipos de projetos e organizações. Assim, ao invés de apresentar um único processo prédefinido, o guia deve indicar várias alternativas para cenários típicos no contexto de MPEs.

Além desses requisitos, o guia deve ser direcionado especificamente para monitoração e controle de projetos de software, considerando suas características e dificuldades específicas [Acuña 00].

\section{Uma abordagem para Modelagem do Processo de Monitoração e Controle}

De acordo com os requisitos identificados, o presente artigo propõe a extensão da abordagem ASPE/MSC para suportar a modelagem de um processo de monitoração e controle de projetos de software, adaptada às características e limitações típicas das MPEs e utilizando um guia alinhado aos modelos de referência para a melhoria da qualidade de software (CMMI-DEV V1.2, MPS-BR e ISO/IEC 15504) [MCT 05].

Para implantar efetivamente qualquer processo em uma organização, é necessário realizar primeiramente o levantamento do processo atualmente utilizado e, em seguida, a modelagem do processo que a organização irá utilizar. Para suportar o estabelecimento de processos de software de forma incremental, a ASPE/MSC é dividida em quatro fases: diagnóstico do processo de software atual, análise estratégica, definição do processo e implantação do processo.

Antes de iniciar a modelagem propriamente dita, na ASPE/MSC é realizada a iniciação, onde a organização se prepara para o programa de melhoria, estabelecendo os objetivos e a política de qualidade, bem como indicando as pessoas que preencherão os papéis no processo. Após a iniciação, é realizado o diagnóstico do processo de software atual por meio de uma avaliação dos processos, levantando os pontos fortes e fracos, bem como estabelecendo as recomendações de melhoria.

A fase seguinte, análise estratégica, dá inicio à modelagem do processo em si. Neste momento, os processos a serem modelados são priorizados e são estabelecidos um ou dois processos que serão modelados no primeiro ciclo de melhoria.

$\mathrm{Na}$ fase de definição dos processos, cada processo escolhido na fase anterior é modelado, refinado e documentado em um guia de processo. Na definição dos processos 
não somente é descrito o que está sendo feito atualmente, mas também são utilizados os modelos de referência de processos quando são encontradas oportunidades de melhoria.

$\mathrm{Na}$ fase final, de implantação, um projeto piloto é escolhido para aplicação do processo modelado, de forma a avaliar a aplicabilidade do modelo de processo resultante e a identificar as possíveis melhorias necessárias.

Desta forma, a ASPE/MSC oferece uma abordagem para estabelecer processos em uma organização. Porém, aplicando a abordagem na prática [Wangenheim 06b] [Hauck 04] [Weber 05], foi observado que este suporte ainda poderia ser melhorado, especificamente considerando o alinhamento de processos aos modelos de referência. Em função disso, o enfoque deste trabalho é integrar à abordagem de modelagem de processo ASPE/MSC, um guia de referência que, na fase de definição dos processos, onde é realizada a modelagem de processo prescritiva [Acuña 00], complemente o modelo descritivo já levantado na organização, alinhando-o aos modelos de referência. O resultado é uma evolução da abordagem ASPE/MSC, apresentada na figura 1, onde as etapas originais da abordagem foram mantidas, mas na etapa de refinamento do processo foi introduzida a utilização do Guia de Processo (de monitoração e controle).

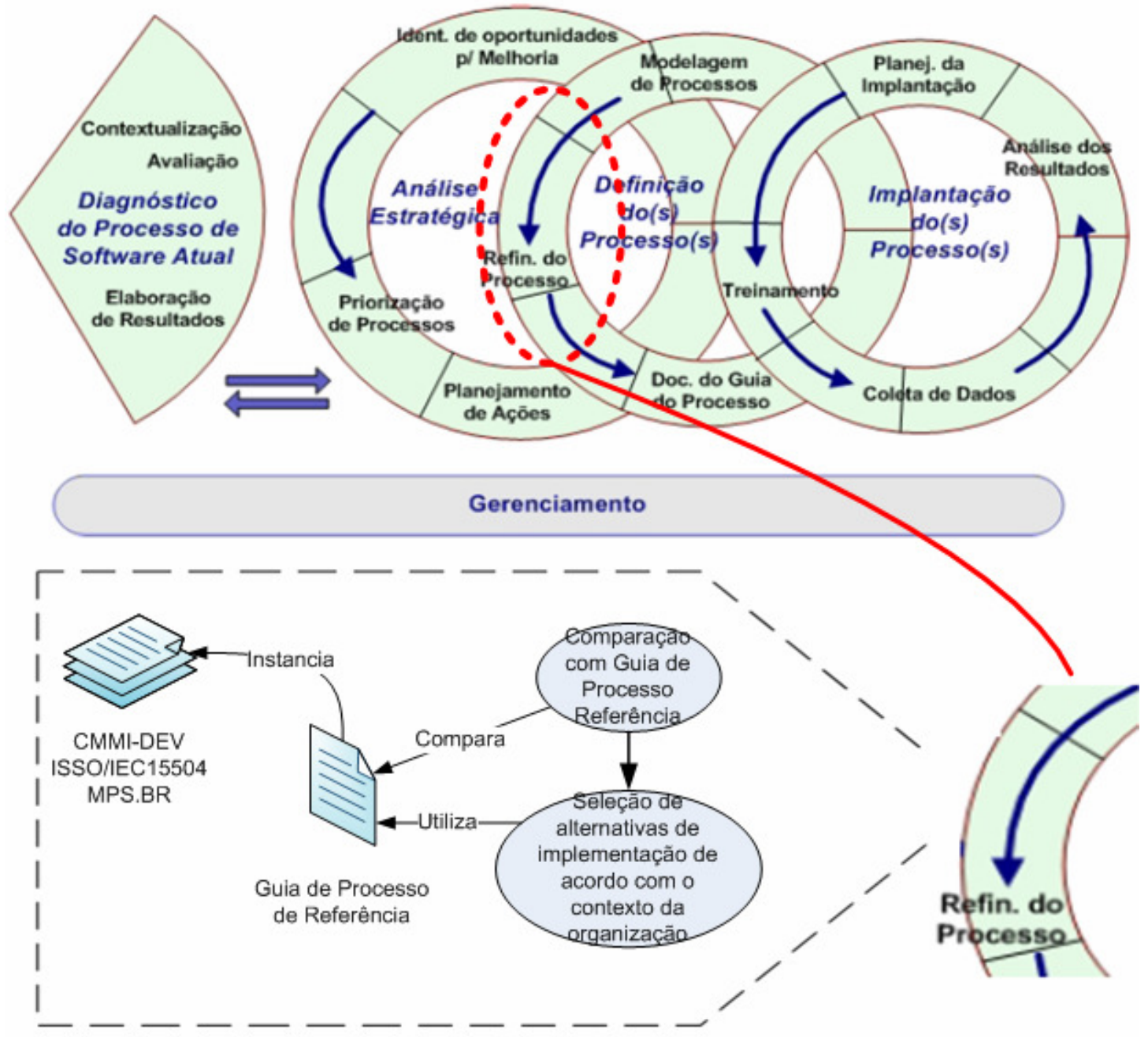

Figura 1: Extensão da abordagem ASPE para a Modelagem de Processo utilizando um guia. Baseado em [Weber 05]. 


\section{Um Guia de Monitoração e Controle de Projetos de Software}

O Guia de Monitoração e Controle de Projetos de Software, no contexto deste trabalho, é utilizado para auxiliar as MPEs a identificarem as atividades nas quais o seu processo atual de monitoração e controle (caso exista) apresenta carências em relação aos modelos e normas de referência. O guia é estruturado por meio de um processo de referência de monitoração e controle adaptado às características de MPEs (vide figura 2 ), seguindo a notação proposta em [Thiry 06]. Este processo define as principais atividades necessárias para o atendimento das práticas e geração dos resultados esperados pelos modelos de referência de melhoria de processo escolhidos.

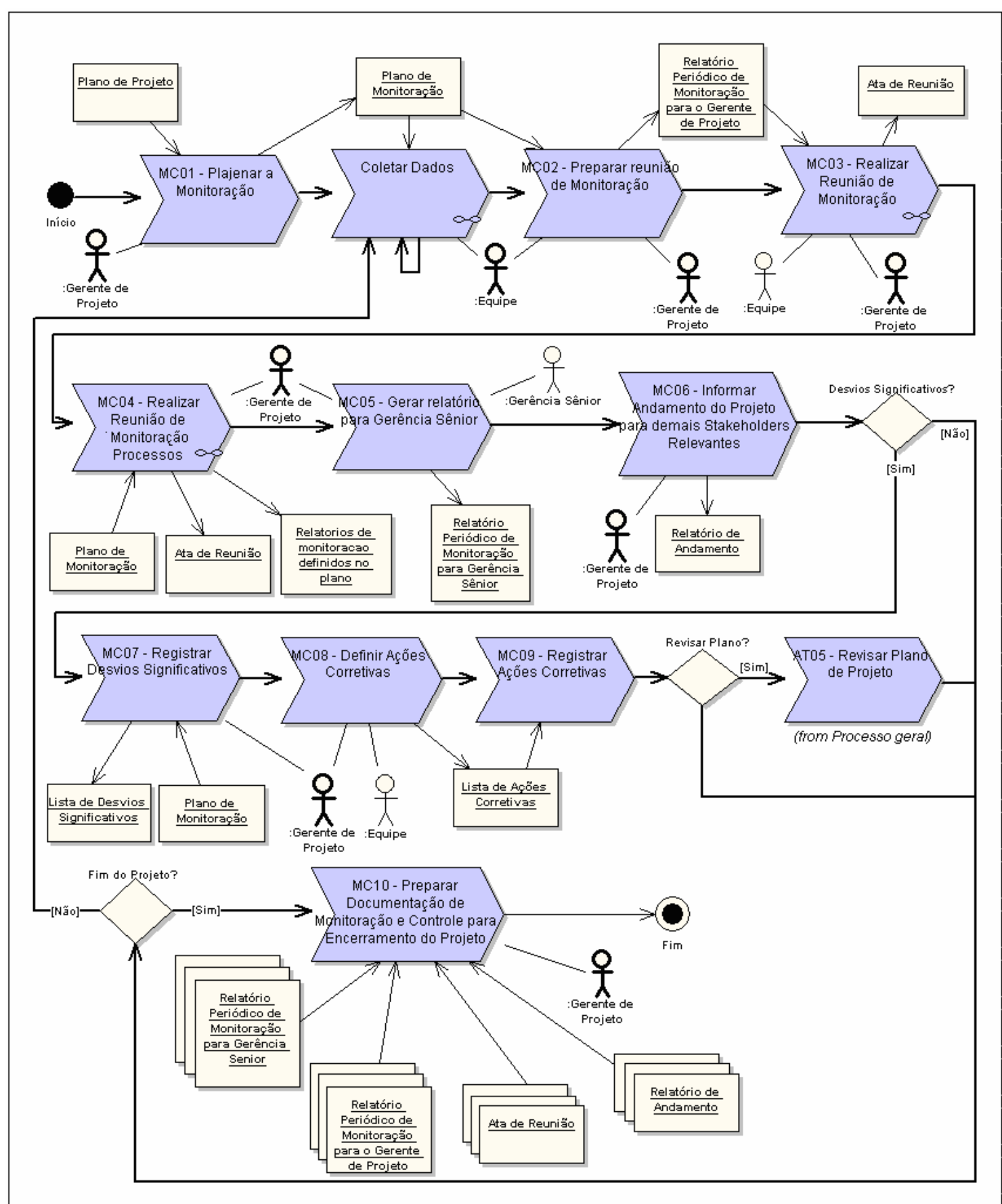

Figura 2: Modelo de Processo de Monitoração e Controle de Projetos. 
Cada uma das atividades do processo de monitoração e controle, mostrada na figura 2, é detalhada no Guia. O detalhamento de cada atividade estende o formato proposto em [Weber 05], adicionando orientações para adaptação da atividade a cada perfil de organização, apresentando alternativas de técnicas e ferramentas possíveis de serem utilizadas na sua execução. Como exemplo, um extrato do detalhamento da atividade MC03 é apresentado na figura 3.

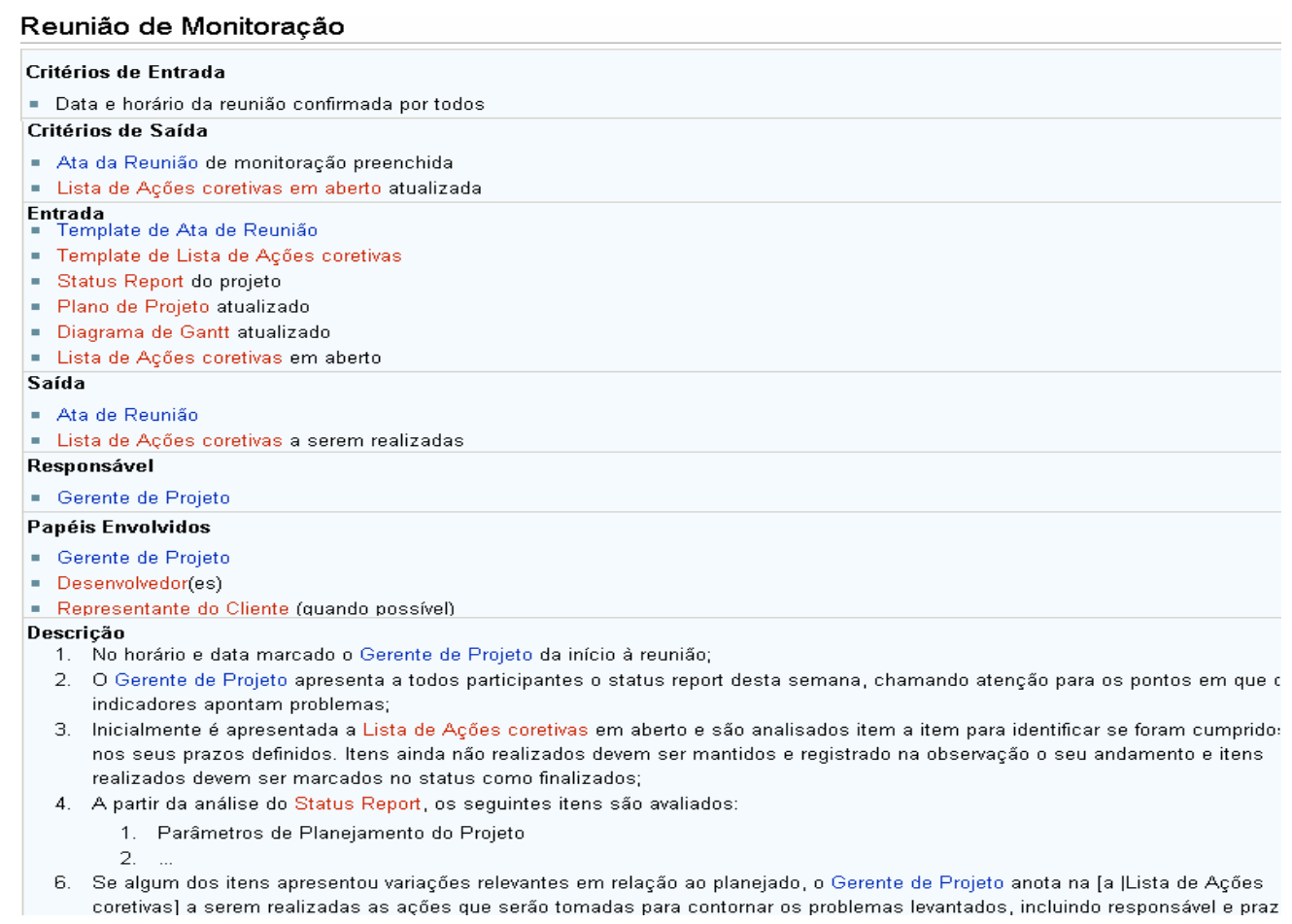

Figura 3: Extrato de um exemplo de descrição de uma atividade.

A descrição de uma atividade inclui:

- Critérios de entrada e saída: são os requisitos que devem ser estar satisfeitos para que uma atividade possa ser iniciada ou finalizada;

- Entrada e Saída: são os artefatos utilizados e gerados pelo processo. São identificados e fornecidos templates de documentos, exemplos de artefatos e cenários de utilização, como por exemplo: conjuntos de indicadores a serem observados para a monitoração. Um exemplo de template de Relatório de Status do Projeto é apresentado na figura 4;

- Ferramentas: indicação de ferramentas (softwares, planilhas, etc.) que possam facilitar ou automatizar a atividade;

- Técnicas: indicação e descrição de técnicas adequadas para serem aplicadas no contexto de projetos de software em MPEs;

- Descrição da atividade: neste item são apresentados os passos que devem ser seguidos para a realização da atividade. São também indicadas as formas de 
utilização das entradas e saídas e como as ferramentas e técnicas devem ser aplicadas no contexto da atividade;

- Cenários: apresentação dos cenários de realização da atividade, simulando a sua realização prática e auxiliando no entendimento e adaptação da atividade;

- Referências: indicação das referências que apresentam o alinhamento da atividade às normas, modelos e abordagens de gerência de projetos (CMMI, MPS.BR, ISO/IEC 15504, PMBOK, etc.);

- Orientações para adaptação: além destes pontos principais, na descrição de cada artefato, ferramenta e técnica, são indicadas orientações para adaptação da atividade ao contexto específico de uma organização. Por exemplo: o valor agregado é uma técnica indicada para auxiliar na monitoração das atividades, entretanto ela somente deve ser utilizada se os gerentes de projeto possuem o conhecimento necessário, caso contrário, antes da utilização destes indicadores, os gerentes de projeto devem ser treinados. Outro exemplo, custos só devem ser observados pelos gerentes de projetos se a política da organização divulga ou pretende divulgar os custos associados a uma tarefa, caso contrário deve ser utilizado somente homens/hora.

- Medidas para monitoração: seguindo o que estabelecem os modelos e normas, as próprias atividades do processo de monitoração e controle também devem ser monitoradas e controladas. Desta forma, para cada atividade, são apresentadas medidas típicas que podem ser coletadas na realização da atividade para possibilitar a sua monitoração.

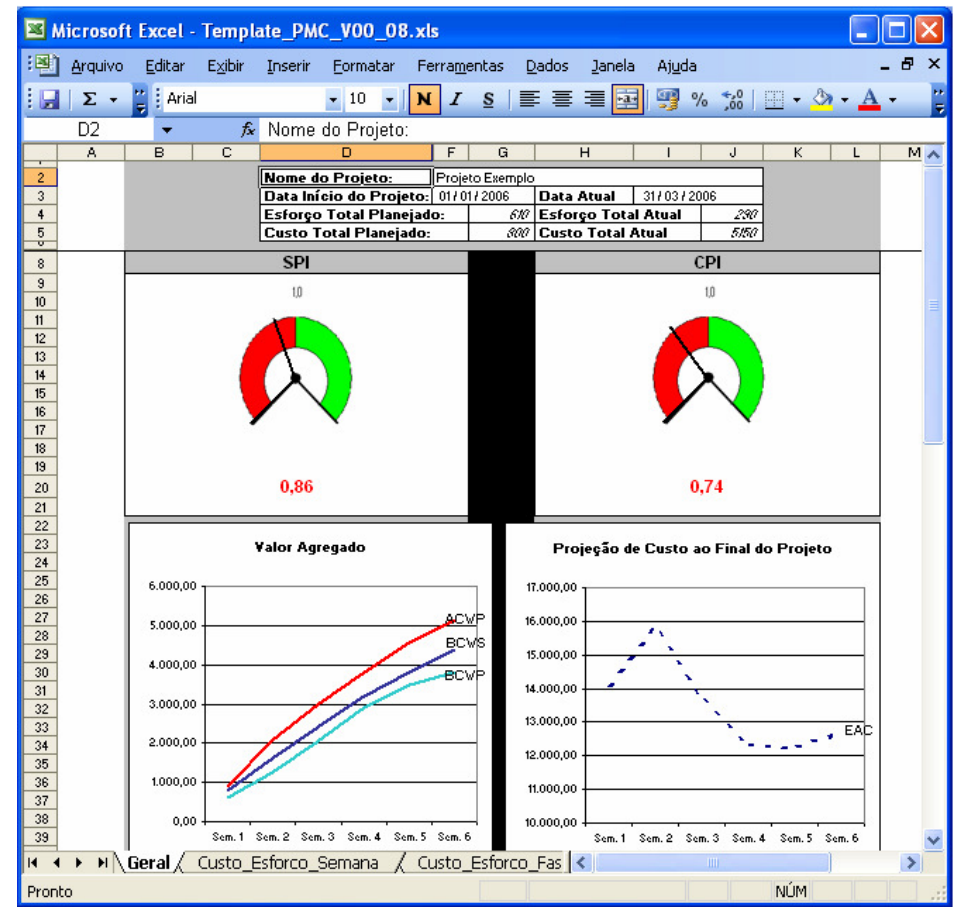

Figura 4: Template de Status Report gerado em planilha eletrônica. 
Para facilitar o acesso e a disseminação, o Guia está sendo desenvolvido para ser exibido em um WIKI [WIKI 07], na forma de um Electronic Process Guide[27] (vide figura 5).

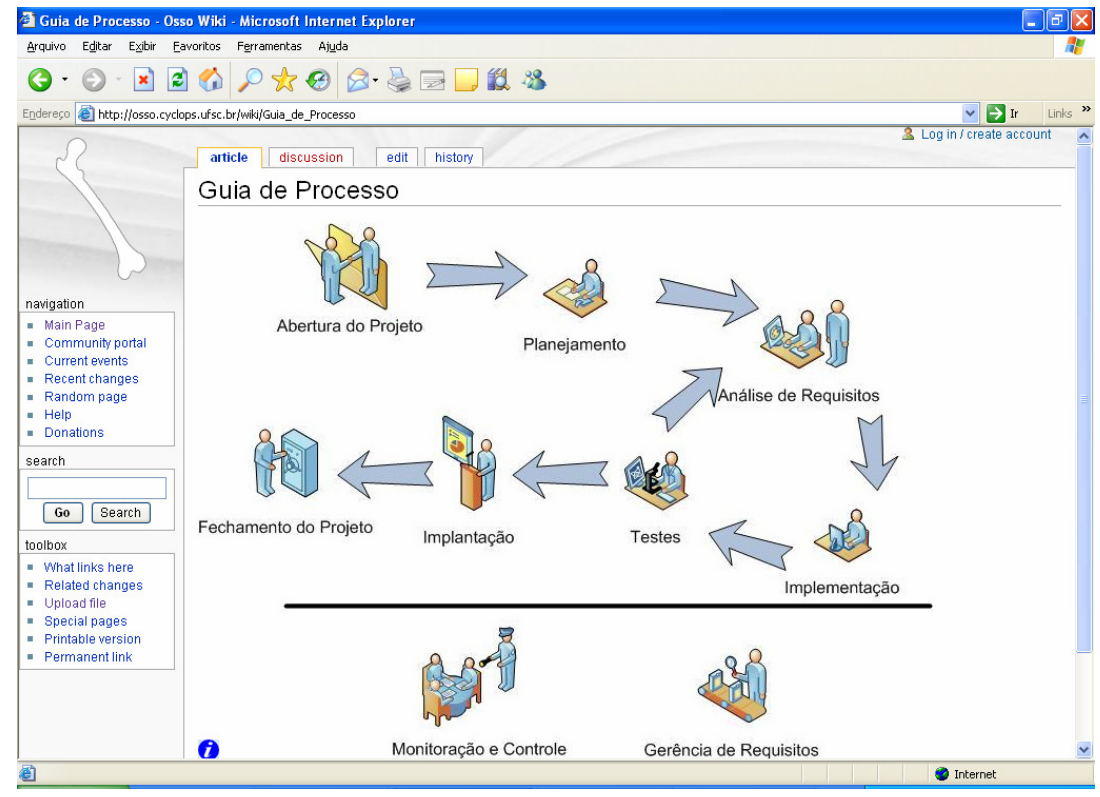

Figura 5: Tela inicial do Guia de Processo no WIKI.

\section{Estudo de Caso: Modelagem do Processo de Monitoração e Controle no CYCLOPS}

Assim que uma primeira versão draft da abordagem foi concluída, foi realizado um primeiro piloto de utilização da abordagem na organização de pesquisa CYCLOPS [CYCLOPS 07], da Universidade Federal de Santa Catarina (UFSC), especializada na pesquisa e desenvolvimento de tecnologias inovadoras na área de processamento de imagens médicas. O CYCLOPS abrange vários laboratórios da UFSC e conta, atualmente, com mais de cinqüenta pesquisadores. Apesar de não se tratar de uma empresa, esta organização de pesquisa foi escolhida como primeiro cenário para estudo de caso pela proximidade e por apresentar características semelhantes a uma MPE: restrição de recursos, processo de software imaturo (de forma geral), poucos níveis hierárquicos, papéis pouco definidos, gerentes de projetos com background técnico, etc. Além deste primeiro piloto, para fortalecer os resultados da aplicação, outros cinco estudos de caso de aplicação da abordagem apresentada neste artigo, já estão planejados para serem realizados em MPEs.

O estudo de caso foi aplicado no contexto de um programa de melhoria que está sendo atualmente implantado no CYCLOPS, com o objetivo de levar a organização de pesquisa ao nível G do MPS.BR. Um dos principais processos enfocado neste nível é monitoração e controle de projetos, incluído no processo de Gerência de Projetos. Durante o programa de melhoria, a abordagem ASPE/MSC foi utilizada para a modelagem de processos. 


\section{Como foi realizado o estudo de caso?}

Inicialmente, preparando a definição do processo de monitoração e controle, foram realizados treinamentos na área de gerência de projetos e especificamente de monitoração e controle de projetos num total de 16 horas. O público alvo foram os gerentes de projeto, com o objetivo de nivelar os conceitos e estabelecer uma compreensão mínima dos respectivos processos.

Após os treinamentos, seguindo a ASPE/MSC, o primeiro trabalho realizado foi a modelagem descritiva do processo, tentando identificar como era realizada a monitoração e o controle informais dos projetos. Para isso, foram identificados os principais grupos de interessados no processo de monitoração e controle: equipes dos projetos, gerentes de projetos e a alta gerência. Como consequiência disto, também se iniciou a definição explícita de um organograma e de descrições de papéis no CYCLOPS, que até então somente existia informalmente.

Num segundo momento, foram levantados os processos atualmente executados pelos gerentes de projetos no CYCLOPS. Como cada um dos gerentes adotava uma forma diferente de monitoração e controle, foram realizados workshops [Thiry 06] com todos os gerentes de projeto e a gerência sênior. Naquela ocasião, todos apresentaram informalmente o seu processo e relataram os principais pontos positivos e negativos observados por eles, além de algumas melhorias que já estavam introduzindo após os treinamentos.

A partir destes relatos, foi montado, durante os workshops, um modelo descritivo do processo de monitoração e controle, seguindo a notação proposta em [Thiry 06]. Este modelo foi documentado pelo engenheiro de processo e revisado pelos gerentes. Para completar o processo, foram discutidas com os gerentes de projeto e representantes da gerência sênior formas de relatos, frequiências etc. Estas discussões foram lideradas por consultores do LQPS/UNIVALI e pelo engenheiro de processo do CYCLOPS.

No final, a equipe de engenharia de processo (consultores e engenheiro de processo do CYCLOPS) revisaram e completaram o modelo de processo em relação ao seu alinhamento ao modelo MPS.BR, tomando como base o draft do guia do processo de referência de monitoração e controle. Cada uma das atividades relacionadas ao processo foi então documentada e detalhada no WIKI da organização e liberada para revisão por todos os gerentes de projeto.

A implantação do processo modelado foi gradativa, iniciando por um projeto piloto de curta duração e, após alguns ajustes, foi sendo estendido para toda a organização. Inicialmente, havia sido previsto que a monitoração seria realizada por meio de planilhas eletrônicas. No entanto, pela dificuldade apresentada no preenchimento destas no projeto piloto, paralelamente foi iniciada a extensão da ferramenta dotProject [dotProject 07]. Esta ferramenta já era adotada para o planejamento de projetos no CYCLOPS e passou a suportar também o processo de monitoração e controle, de acordo com o processo definido, conforme demonstra a figura 6 . 


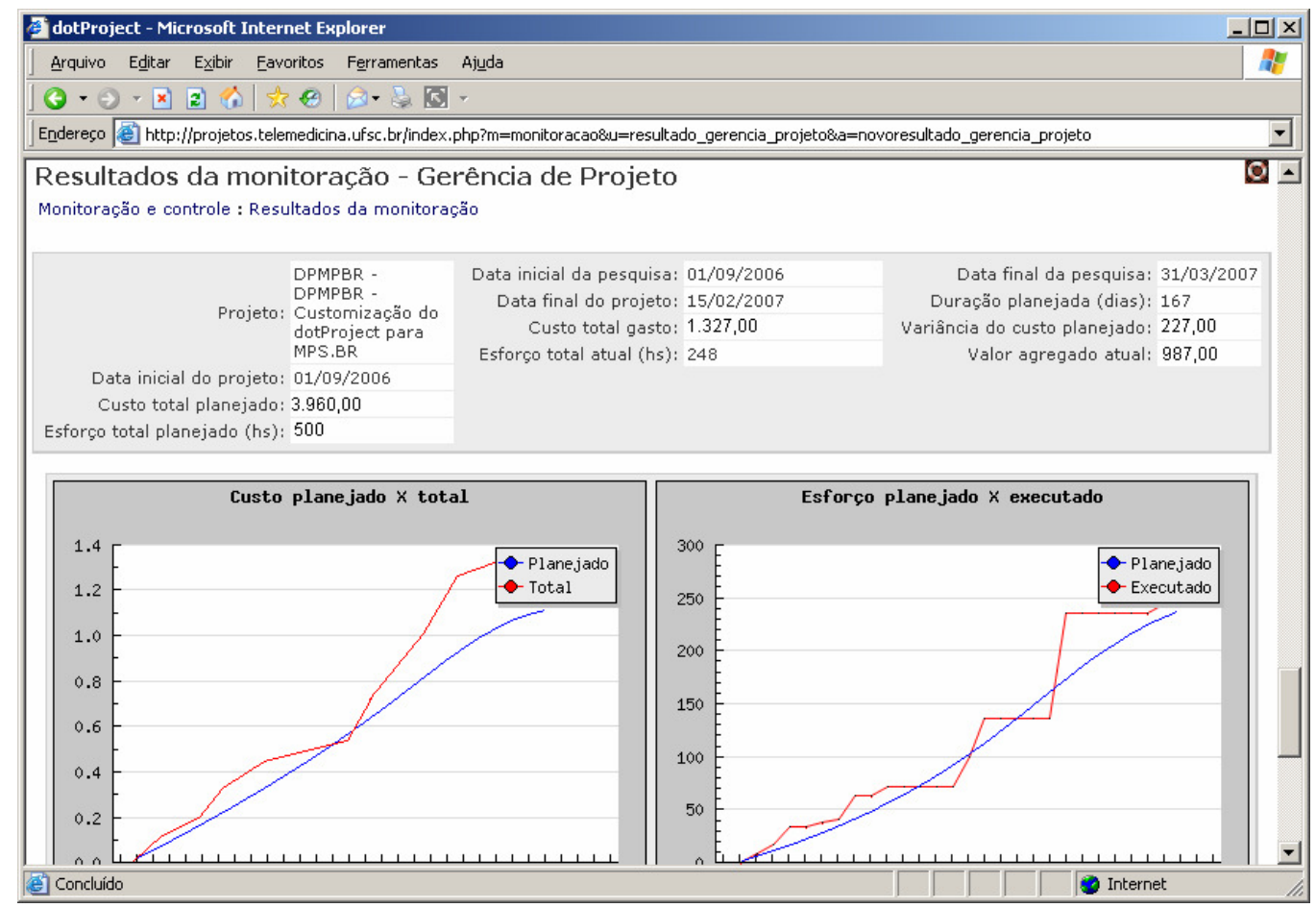

Figura 6: Módulo de Monitoração e Controle no dotProject.

Atualmente o processo de monitoração e controle está sendo adotado em todos os projetos executados pela organização, que tenham sido iniciados após a data da publicação da primeira versão do processo no WIKI.

\section{Primeiros resultados observados}

A partir da realização deste primeiro estudo de caso de utilização da abordagem proposta, foram observados os primeiros indícios de que o estabelecimento de um processo pode ser facilitado utilizando uma abordagem que inclui um guia de processo de referência.

Foram coletados os dados referentes a custos (homens/hora) empregados na modelagem do processo de monitoração e controle apoiado pelo guia. Durante os três meses em que foi realizada a modelagem do processo de monitoração e controle, foram empregados 265 homens/hora na elaboração do modelo e 80 homens/hora para o detalhamento das atividades do processo. Conforme mostra a figura 6, comparando-se o esforço total disponível no CYCLOPS (somando todas as horas trabalhadas de todos os pesquisadores), ao percentual empregado no detalhamento do processo (onde o guia é utilizado), no mesmo período, o resultado é de $2 \%$. Já o esforço percentual total empregado na modelagem foi de $9 \%$ em relação ao total de esforço disponível.

O comparativo do esforço empregado em relação ao esforço total disponível é demonstrado na figura 7. 


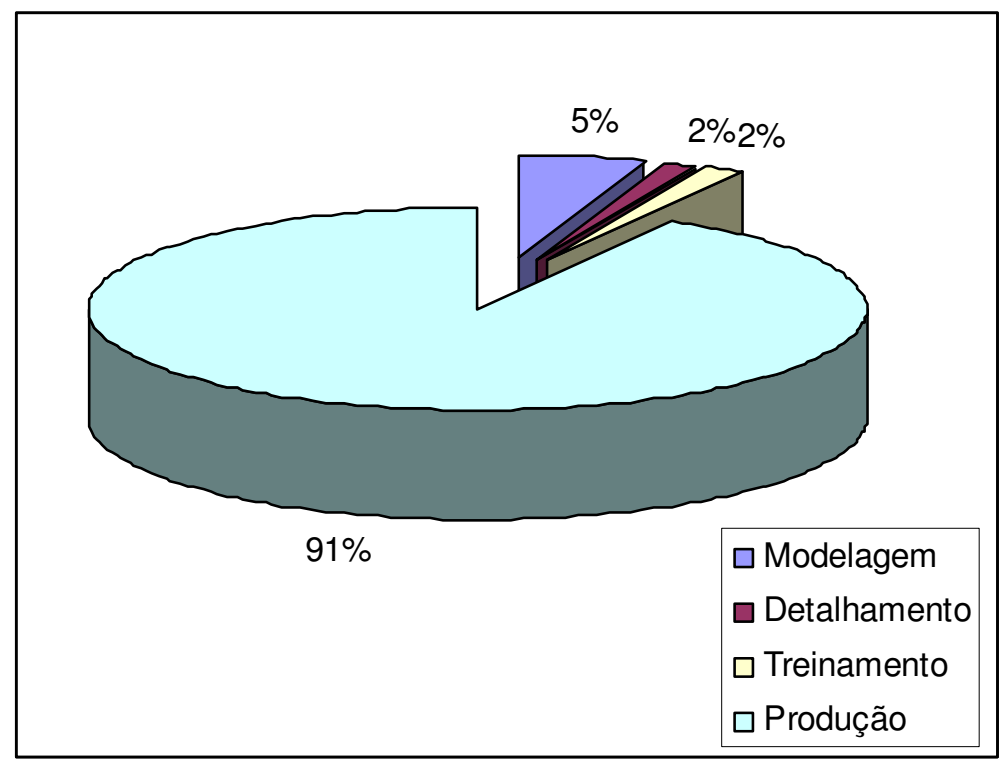

Figura 7: Comparativo do esforço empregado na modelagem.

Como a aplicação do processo de monitoração e controle de projetos no CYCLOPS é recente, ainda não foi possível obter informações quantitativas de melhoria do processo e do produto. Entretanto, a partir de uma pesquisa qualitativa, onde foram realizadas 20 entrevistas, foi observado que os entrevistados, em geral (75\%), percebem a melhoria do processo de monitoração e controle em termos de visibilidade do processo. Por outro lado, $70 \%$ percebem que o trabalho aumentou em função da introdução de maior formalismo na coleta de dados para a monitoração, que antes era realizada de maneira informal.

\section{Discussão e Conclusão}

Neste trabalho é apresentada a evolução da abordagem ASPE/MSC para modelagem de processos em MPEs, complementando-a pela inclusão de um guia de monitoração e controle de projetos. A utilização do guia visa facilitar a complementação do modelo descritivo, alinhando-o aos principais modelos e normas de melhoria.

Considerando os requisitos identificados para um guia, pode-se observar que a utilização do guia de monitoração e controle de projetos atende os requisitos identificados, oferecendo assim maior suporte neste contexto do que outras abordagens, modelos ou normas existentes. Também a sua primeira aplicação prática dá indícios do seu beneficio.

Com base nos resultados obtidos, o guia está sendo completado, principalmente pela coleta de experiências próprias e da literatura sobre aplicações do processo de monitoração e controle em MPEs, para ampliar o suporte e diversificar as alternativas oferecidas.

Como mais um resultado desta pesquisa também está sendo implementada uma extensão da ferramenta dotproject adaptada ao processo de referência de monitoração e controle definido no guia. 


\section{Referências}

ACUÑA, A., FERRE X., López M., Mate L.The "Software Process: Modeling, Evaluation and Improvement”, Argentina: World Scientific Publishing Company, 2000.

ALEGRIA Julio Ariel Hurtado, Maria Cecilia Bastarrica "Implementing CMMI using a Combination of Agile Methods", Clei - Latinamerican Center for Informatics Studies - Electronic Journal, Vol. 9 Num. 1 Pap. 7: June 2006.

ANSI/EIA 748, “A Standard for Earned Value Management Systems”, ANSI, 1998.

BECK Kent, "Extreme Programming Explained: Embrace Change”, Addison-Wesley, 1999.

BECKER-Kornstaedt Ulrike, "Towards Systematic Knowledge Elicitation for Descriptive Software Process Modeling”, IESE-Report No. 036.01/E Version 1.0 September 2001

CYCLOPS, Disponível em: <www.cyclops .ufsc.br>, Acesso em: 01/04/2007.

DAFT R.L., “Organisation Theory and Design”, 4th ed., West Publishing, 1992.

DOTPROJECT, disponível em <www.dotproject.net>. Acesso em: 23 mar. 2007.

GALORATH Mike Ross Galorath Inc. "Integrating Three Level 2 CMMITM Process Areas: Closing the Loop on Software Project Management" IEEEAC paper 1410, Revision B, December 19, 2005

HAUCK Jean Carlo Rossa, Christiane G. Wangenheim, "Modelando o Processo de Software em uma Pequena Empresa - O Caso VOID CAZ", VI Simpósito Internacional de Melhoria de Processos de Software, São Paulo, 2004.

HUGHES B., M. Cotterell, "Software Project Management”, 3rd Edition, McGraw-Hill, 2002

ISO/IEC - International Organization for Standardization., "ISO/IEC 15504: Information Tecnology Process Assessment, Part 1 to Part 5", ISO/IEC International Standard, 2005.

ISO/IEC - International Organization for Standardization. "ISO/IEC 10006: Quality Management Guidelines to Quality in Project Management”, ISO/IEC International Standard, 1997.

JALOTE Pankaj, "CMM in Practice: Processes for Executing Software Projects at Infosys", Addison Wesley Longman, 2000.

JOHNSON D.L. and J.G. Brodman, "Tailoring the CMM for Small Businesses, Small Organizations, and Small Projects, Elements of Software Process Assessment and Improvement, K. El Emam and N.H. Madhavji, eds., IEEE CS Press, 1999, pp. 239-259.

KULPA Margaret K., Kent A. Johnson, "Interpreting the CMMI®: a process improvement approach", Auerbach Publications, 2003.

MACHADO, Luiz Felipe Dionísio Cavalcanti. "Modelo para Definição de Processos de Software na Estação TABA”. Rio de Janeiro, 2000.

MARTINS José Carlos Cordeiro, "Gerenciando Projetos de Desenvolvimento de Software com PMI, RUP e UML”, Brasport, 3. Ed, Rio de Janeiro, 2006.

MCT - Ministério da Ciência e Tecnologia. "Qualidade e Produtividade no Setor de Software Brasileiro, Resultados da Pesquisa 2005” (resultados antecipados recebidos por e-mail do MCT).

MD - Ministério do Desenvolvimento, Indústria e Comércio Exterior, Secretaria do Desenvolvimento da Produção, Departamento de Micro, Pequenas e Médias Empresas, "Definições e Estatísticas Internacionais”, Brasília, 2002.

MURCH R., “Project Management: Best Practices for IT Professionals”, Prentice-Hall, 2000

OMG - OBJECT MANAGEMENT GROUP. "SPEM: Software Process Engineering Metamodel", version $\quad$ Object $\quad$ Management Group <http://www.omg.org/technology/documents/formal/spem.htm>. Acesso em 01 abr. 2007. 
PAULK M.C., "Using the Software CMM in Small Organizations,"Proc. Joint 16th Pacific Northwest Software Quality Conf. and 8th Int'1 Conf. Software Quality, 1998, pp. 350-360.

PIKKARAINEN Minna and Annukka Mäntyniemi, "An Approach for Using CMMI in Agile Software Development Assessments: Experiences from Three Case Studies", SPICE 2006 conference, Luxemburg, May 2006.

PMI - Project Management Institute, "Um Guia do Conjunto de Conhecimentos em Gerenciamento de Projetos Terceira edição (Guia PMBOK®)”, PMI, Pennsylvania, 2004.

ROUT T.P. et al., “The RAPID Assessment of Software Process Capability,” Proc. 1st Int'1 SPICE Conf., Centre for Software Eng., Dublin City Univ., 2000, pp. 47-56.

SCOTT Louise, Jörg Zettel, Dirk Hamann. "Suporting Process Engineering in Pactice: An Experience Based Scenario", Fraunhofer Institut Experimentelles Software Engineering, Kaiserslauten, 2000.

SCOTT Louise, Stalhane Tor. "Experience Repositories and the Postmortem”. School of Computer Science and Engineering,University of New South Wales, Australia, 2003.

SCHWABER Ken, “The Scrum development process”, In OOPSLA '95 Workshop on Business Object Design and Implementation, Austin, Texas, USA, ACM Press, October 1995.

SEBRAE, Disponível em: <www.sebrae.com.br>. Acesso em: 23/03/2007.

SEBRAE-SP, "Pesquisa e Planejamento Estratégico. Relatório de Pesquisa: MPEs de Base Tecnológica: Conceituação, formas de financiamento e análise de casos brasileiros”, São Paulo, 2000.

SEI - Software Engineering Institute, "Capability Model Integration, version 1.2 - CMMI® for Development, Version 1.2, 2006.

SIS - Software Industry Statistics for 1991-2005, Enterprise Ireland, 2006.

SOFTEX , "MPS.BR - Melhoria de Processo do Software Brasileiro”, Guia Geral, Versão 1.1, 2006.

STOREY D.J., "Entrepreneurship and the New Firm”, Croom Helm, 1982.

THIRY Marcello, Christiane Gresse von Wangenheim, Alesandra Zoucas, Kênia Pickler, "Uma Abordagem para a Modelagem Colaborativa de Processos de Software em Micro e Pequenas Empresas", V Simpósio Brasileiro de Qualidade de Software - SBQS`2006.

WANGENHEIM C. Gresse von, Ita Richardson, "Why Are Small Software Organizations Different?", IEEE Software, vol. 24, no. 1, 2007, pp. 18-22.

WANGENHEIM C. Gresse von, A. Anacleto, and C.F. Salviano, "Helping Small Companies Assess Software Processes,” IEEE Software, vol. 23, no. 1, 2006, pp. 91-98.

WANGENHEIM Christiane G., Jean Carlo R. Hauck: "Experiences on establishing software processes in small companies". Volume 48, Issue 9 , September 2006, Pages 890-900.

WEBER Sérgio, “ASPE / MSC: Uma Abordagem para Estabelecimento de Processos de Software em Micro e Pequenas Empresas", Dissertação de Mestrado, Universidade Federal de Santa Catarina, 2005.

WEBER Sérgio, Christiane Gresse von Wangenheim, Jean Carlo R. Hauck, "Estabelecendo Processos de Software em Micro e Pequenas Empresas", Simpósio Brasileiro de Qualidade de Software, Porto Alegre, 2005.

WIKI, Disponível em: <www.wiki.org>. Acesso em: 13/04/2007. 